\title{
A simple design rule for 1 st order form-closure of underactuated hands
}

\author{
S. Krut ${ }^{1}$ and V. Bégoc ${ }^{1, *}$ \\ ${ }^{1}$ LIRMM (CNRS \& Univ. Montpellier 2), Montpellier, France \\ "now at: B+ Equipment, Gemenos, France
}

Received: 27 February 2010 - Revised: 30 June 2010 - Accepted: 19 August 2010 - Published: 8 February 2011

\begin{abstract}
The property of form-closure of a grasp, as generally defined in the literature, is based on the assumption that contact points between the hand and the object are fixed in space. However, this assumption is false when considering a grasp exerted by an underactuated hand, since in this case, it is not possible to control the position of each phalanx independently. In spite of researchers' interest in studying form-closure, none of the available published work on this subject takes into consideration the particular kinematics of underactuated hands. Actually, there are few available tools to qualify or quantify the stability of a grasp exerted by an underactuated hand, thus the design of underactuated hands mostly results from an intuitive approach. This paper aims to reduce this gap.
\end{abstract}

A classification of underactuated hands is proposed, based on the expression of contact forces. This highlights the influence of non-backdrivable mechanisms introduced in the transmission of the closing motion of the hand on the stability of the grasp. The way to extend the original definition of form-closure to underactuated grasps is illustrated. A more general definition is formulated, which checks the stability of the set "object + hand". Using this new definition, a simple rule is proposed for designing a hand capable of achieving 1st order form-closed grasps.

This paper was presented at the IFToMM/ASME International Workshop on Underactuated Grasping (UG2010), 19 August 2010, Montréal, Canada.

\section{Introduction}

The concept of underactuation makes it possible to create grippers which automatically adapt to the geometry of the grasped object, without requiring a large number of sensors or actuators, nor a complex control strategy. Thus, using a simple binary control, such as the one usually used to drive the closing/opening motion of a parallel-jaw gripper, an underactuated gripper permits to increase the number of contact points, resulting in an enveloping grasp that should be, a-priori, more robust than a two-contact grasp. However, underactuated grippers are rarely used in the industry, first because most of gripping operations can be achieved using simple grippers dedicated to unique objects, but also because, in certain cases, underactuated grippers exhibit aberrant behavior which leads to an unstable grasp.
In Laliberté et al. (2002), a characteristic phenomenon of underactuated hands, the ejection phenomenon, is described. In certain configurations of the finger, the distribution of contact forces degenerates, i.e. some phalanxes must exert a negative force on the object in order to guarantee static equilibrium of the finger. Since a contact action is unidirectional, equilibrium cannot be attained and the ejection of the object from the hand is initiated. The ejection phenomenon highlights the need for a more in-depth investigation of the stability of a grasp exerted by an underactuated hand, considering the particular kinematics of this type of mechanism. Indeed, there has been little work dedicated to studying the stability of a grasp exerted by an underactuated hand. There exist then only few tools for designing such an underactuated hand in order to maximize its ability to stabilize an object. As a result, their design often results from an intuitive approach. 
There are two main criteria which can be used to characterize the robustness of a grasp: form-closure and forceclosure. These two properties permit to perform a local and static study of the stability of a grasp. Form-closure describes the capability of a hand to prevent any motion of a grasped object. Force-closure describes the capability of a hand to counterbalance any external disturbances exerted on a grasped object, by applying a combination of contact forces which respect the capabilities of actuators and the condition of friction (Bicchi, 1995). In contrast to force-closure, formclosure is a purely geometric property since it does not depend on actuators capability nor on the eventual presence of frictional forces between the gripper and the object.

These two properties have been the subject of many investigations but, to the best of our knowledge, these have never been extended to the particular case of underactuated grasps $^{1}$ (even work in Luo et al. (2004) on analysis of underactuated grasps does not deal with the particular kinematics of underactuated hands). This paper focuses on form-closure and aims to propose a simple design rule for 1 st order formclosure of underactuated hands. It is based on some preliminary work introduced in Bégoc et al. (2006) and Krut et al. (2010).

\section{Form-closure}

\subsection{Original definition}

Form-closure can be described as the capability of a hand, or more generally of a set of constraining contacts, to prevent motion of a grasped object. The following definition is used for form-closure, a definition which is largely accepted in the literature:

"A grasp is said to be form-close if, and only if, for every motion of the object, at least one contact constraint is violated." (Bicchi, 1995)

The term "contact constraint" relates to the fact that the relative motion of two solid bodies in contact is constrained by the condition of non-interpenetration. This contact constraint is generally approximated to 1 st order for sake of simplicity. In the major part of the literature, the configuration of the hand is assumed to be fixed, which permits to avoid the kinematic study of the hand. This assumption implies that the control position of each contact phalanx is infinitely rigid and that motors are oversized in comparison with any disturbances likely to be exerted on the object.

\subsection{1 st and 2nd order form-closure}

A distinction must be made between two definitions: 1st order form-closure and 2nd order form-closure (Rimon and

\footnotetext{
${ }^{1}$ As an abuse of language, a grasp realized by an underactuated mechanism is referred to as an underactuated grasp.
}

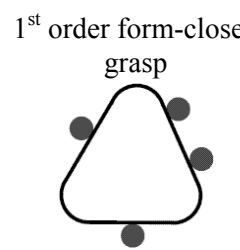

(a)

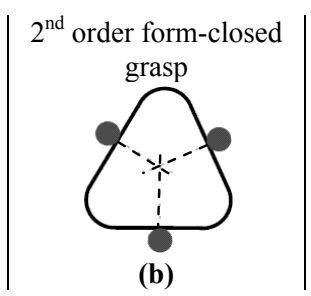

(b)

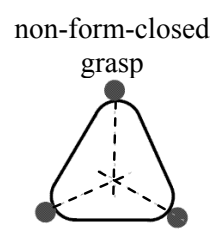

(c)
Figure 1. Illustration of grasp types (figures taken from Cheong (2006)): in situation (a) the object is totally immobilized, in situation (b) it can move with an infinitesimal rotation around the point of concurrency of the contact normals, in situation (c) the object is free to rotate around that point. This phenomenon can be modeled considering the local curvatures of the contact surfaces as explained in Van der Stappen et al. (2000).

Burdick, 1996). This is illustrated using the three grasps depicted in Fig. 1, where the object to be grasped remains the same but where the locations of contact points differ. In all three situations, contact points prevent the object from translating. However, if they also prevent it from rotating in case (a), they do not in cases (b) and (c): the object is free to rotate around the point of concurrency of the contact normals. After an infinitesimal rotation, the object is stopped from rotating in case (b), whereas it is free to escape in case (c). This phenomenon can be modeled considering the local curvatures of the contact surfaces as explained in Carrozza et al. (2005). Grasp (a) is 1st order formclosed, because a 1st order modeling of the grasp is sufficient to demonstrate form-closure, whereas grasp (b) is 2 nd order form-closed, because 2 nd order modeling is required for demonstrating form-closure.

\section{Underactuation}

\subsection{Definition}

"A mechanism is said to be underactuated, when it has fewer actuators than degrees of freedom."

(Birglen et al., 2008)

An actuator is a sub-assembly which causes relative motion between the parts to which it is attached in response to a signal (IFToMM, 2010). The number of degrees of freedom of a system is defined by the number of independent generalized coordinates required to define completely the configuration of a system at any instant of time (IFToMM, 2010). Hence, determining the number of degrees of freedom (dof) of a hand requires considering the coupling that is often introduced between the rotations of interphalanx joints. This is the case for several bionic hands, such as the DLR hands (Butterfass et al., 2001), the Robonaut's hand (Ambrose et al., 2000), the NAIST hand (Ueda et al., 2005) and the hand of humanoid robot HRP-3 (Kaneko et al., 2007). 
The Grübler-Kutzbach equation cannot be applied starightforwadly to determine the degree of freedom of an underactuated hand (Krut, 2005). In fact, this method involves determining the order of a system of kinematic linear equations and it is therefore not applicable to underactuated hands which:

- use unidirectional mechanisms (non-backdrivable mechanisms or anti-return mechanisms) in the transmission of the opening/closing motion of the fingers, because these are modeled using inequalities. This is the case for many underactuated hands such as the Barrett Hand (Townsend, 2000) and the SARAH Hand (Lopez, 2001),

- use compliant bodies to transfer the motor's torque to the fingers as is the case of the RTR II hand (Massa et al., 2002), because the classical system of kinematic equations does not hold anymore.

\subsection{Classification of underactuated hands}

There is a large diversity of mechanical devices which enable a hand to adapt to the geometry of an object. In this paper, we propose to classify underactuated hands based on the expression of contact forces as a function of the torques exerted by the actuators on the mechanism. Hence, an underactuated hand can be:

1. differential,

2. compliant,

3. self-locking,

4. or have a combination of these three properties.

\subsubsection{Differential underactuation}

The first category can be characterized by the formulation given in Krut (2005). A mechanism is said to be differential when its behavior, from a kinetostatic point of view, can be described by the following equations:

$F_{\mathrm{a}}=F_{1}^{\mathrm{a}} r_{1}=\cdots=F_{n}^{\mathrm{a}} r_{n}$

$\theta_{a}+\sum_{i=1}^{n} \frac{\theta_{i}^{\mathrm{a}}}{r_{i}^{\mathrm{a}}}=0$

where $F_{\mathrm{a}}$ is the force, or the actuation torque, exerted on the input of the differential mechanism and $F_{i}^{\mathrm{a}}$ is the force or torque transmitted to the output $i .\left(\theta_{\mathrm{a}}, \theta_{1}^{\mathrm{a}}, \cdots, \theta_{n}^{\mathrm{a}}\right)$ describes the configuration of the differential mechanism, $\left(\theta_{\mathrm{a}}, \theta_{1}^{\mathrm{a}}, \cdots, \theta_{n}^{\mathrm{a}}\right)$ the joint velocities and $\left(r_{1}^{\mathrm{a}}, \cdots, r_{n}^{\mathrm{a}}\right)$ the transmission ratios. A review of the state of the art of differential mechanisms used in underactuated grippers can be found in Birglen and Gosselin (2006).

\subsubsection{Compliant underactuation}

A mechanism is said to be compliant when the forces exerted on its output can be written as a function of the configuration of the mechanism and the stiffness of the mechanical elements introduced into the transmission of the closing motion of the hand:

$\left[\begin{array}{llll}F_{\mathrm{a}} & F_{1}^{\mathrm{a}} & \cdots & F_{n}^{\mathrm{a}}\end{array}\right]^{T}=\boldsymbol{R}\left[\begin{array}{llll}\theta_{\mathrm{a}} & \theta_{1}^{\mathrm{a}} & \cdots & \theta_{n}^{\mathrm{a}}\end{array}\right]^{T}$

where $\mathbf{R}$ is the stiffness matrix of the mechanism.

\subsubsection{Self-locking underactuation}

The category of self-locking underactuated mechanisms is introduced in order to characterize hands which have unidirectional mechanisms in the transmission of the fingers' closing motion. Indeed, this has a strong influence on the expression of contact forces and, as a consequence, on the characteristics of the grasp since this type of mechanisms prevents all return motion of the fingers or phalanxes whatever the disturbances exerted on the grasped object. Theoretically, the contact forces could then be infinite. We define a mechanism to be self-lockable, when the configuration of the mechanism is constrained by a set of inequalities of the type:

$\delta_{i} \theta_{i}^{\mathrm{a}} \geq 0, \forall i=\{1, \cdots, n\}$

$\delta_{i}= \pm 1$ depending on the case.

\subsection{Classification of unidirectional mechanisms}

The unidirectional mechanisms, on which the self-locking hands are based, provide unidirectional transmission of motion, which can be modeled by:

$\theta_{\text {out }} \geq 0$

where $\theta_{\text {out }}$ represents the output velocity of the mechanisms. Unidirectional mechanisms can be spited in two main categories: (1) anti-return mechanisms and (2) non-backdrivable mechanisms.

\subsubsection{Anti-return mechanisms}

Among the available anti-return mechanisms are:

- blocking mechanisms by obstacle, such as the "pawl and ratchet" mechanism or the sliding gear,

- locking mechanisms by bracing or wedging such as a freewheel (with ramp or cam), wrap spring couplings or sliding/bracing mechanisms, such as those used in the underactuated hand designed by Lopez (2001),

- a non-return valve when fluid energy is used. 
In the case of the free-wheel mechanism and wrap spring coupling, the unilateral condition is described differently:

$\theta_{\text {out }} \geq \theta_{\text {in }}$

where $\theta_{\text {out }}$ and $\theta_{\text {in }}$ are the output and input velocities of the mechanism respectively. This condition is identical to condition (5) when the input velocity is zero.

\subsubsection{Non-backdrivable mechanisms}

Non-backdrivable mechanisms provide also the same unilateral condition (5). A mechanism is said to be nonbackdrivable when it is incapable of transmitting motion and power from the output to the input (IFToMM, 2010), as for instance:

- the "triangular wedge" mechanism,

- The "wheel and worm drive" mechanism,

- The "rack and worm drive" mechanism,

- The "lead screw and nut" mechanism.

A non-backdrivable mechanism operates differently from an anti-return mechanism. Nevertheless, it is possible to impose a unidirectional transmission of motion $\theta_{\text {out }} \geq 0$ by maintaining a positive or null force on the input of the nonbackdrivable mechanism.

In practice, it is noticeable that designers of underactuated hands prefer to introduce non-backdrivable mechanisms. The advantage of these mechanisms is that it is sufficient to invert the direction of rotation at the input in order to invert the sense of rotation at the output, and hence to enable opening of the gripper. In the case of a unidirectional mechanism, a mechanism is required to deactivate the anti-return function, as for instance the solution proposed in Lopez (2001) to deactivate a sliding/bracing mechanism.

\section{Extension of form-closure to underactuated hands}

The definition of form-closure, as it is usually encountered in the literature, is based on the assumption that contact points are fixed in space. When considering a robotic hand, this assumption implies to consider that the position control of each phalanx is infinitely rigid, so that the configuration of the hand remains unchanged whatever the amplitude of disturbances exerted on the object. This greatly simplifies the study of the grasp, by avoiding the kinematic study of the hand. This assumption is justified for the majority of robotic hands, for which the position of each phalanx can be independently controlled. In contrast, it is not possible to independently control the position of each phalanx when using an underactuated hand. The assumption of fixed contact points is then false.

In Krut et al. (2010), the definition of form-closure was reformulated so that it applies to the case of underactuated

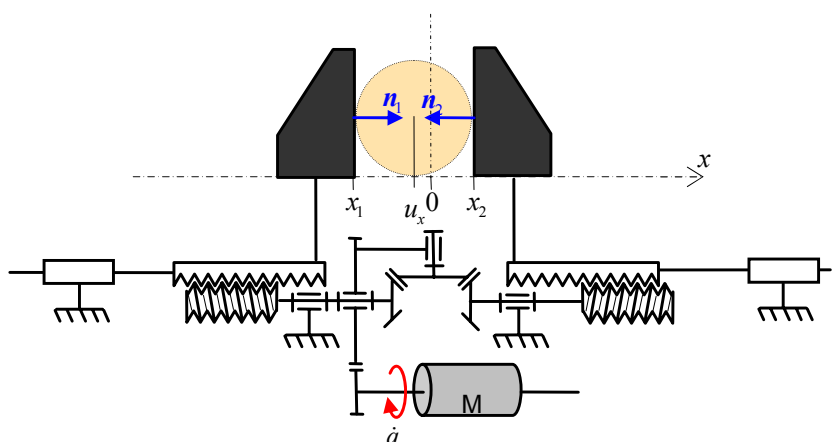

$\dot{q}_{m}$

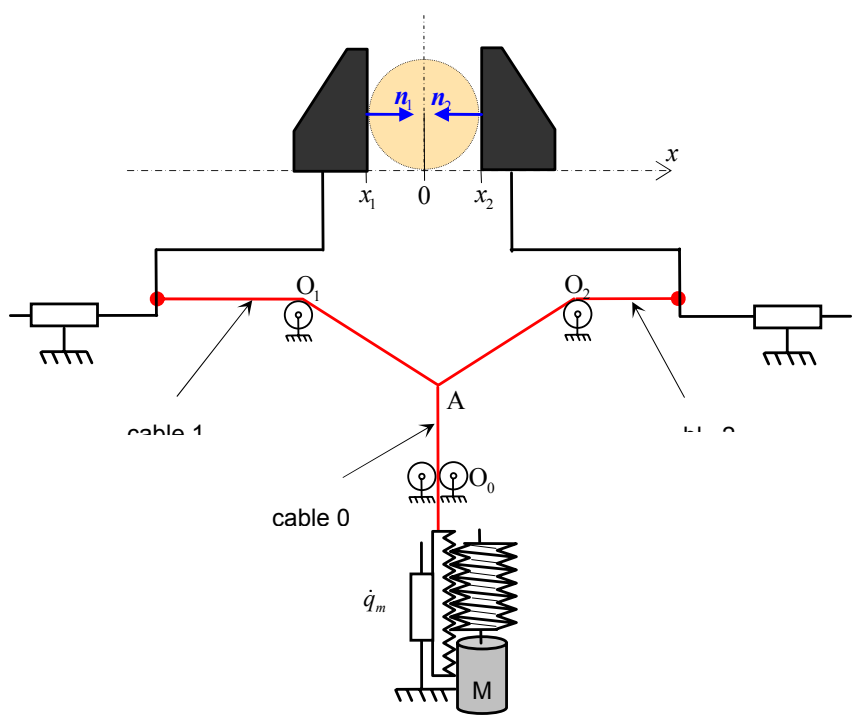

Figure 2. Diagram representing a two contact grasp.

grasps. Since immobility of contact points is not guaranteed, not only the motions of the object have to be studied but also the motions of the grasp, i.e. the motions of the overall system including the object and the hand. Thus, checking form-closure of an underactuated grasp now requires verifying that any variation in the configuration of the grasp is prevented by a set of unilateral kinematic constraints, i.e. the contact constraints and the constraints imposed by the non-backdrivable mechanisms. The definition of form-closure was reformulated as follows:

"A grasp is said to be form-closed if, and only if, for any variation of the configuration of the grasp at least one of the unilateral kinematic constraints is violated."

To illustrate this definition, two simple cases of two-finger grasps are considered (see Fig. 2). These grasps differ in terms of type of transmission used to produce the closing motion of fingers. In order to simplify this analysis, in a way that will make it intuitive, an operational space of dimension one only is considered, i.e. only the translation of the object along $\mathrm{x}$ is studied. 


\subsection{Mechanism A}

The first mechanism is underactuated by a geared differential mechanism (Fig. 2a). The closing motion of each jaw is transmitted via a "wheel and worm drive" non-backdrivable mechanism. Once the two jaws are in contact, they cannot move back, meaning that the object is completely immobilized. The grasp is form-closed.

\subsection{Mechanism B}

The second mechanism is underactuated by a "pulley/tendon" differential mechanism. A single actuator drives the closing motion of the gripper by tightening cable 0 . This tension is transmitted to cables 1 and 2 which are connected to cable 0 at point A (Fig. 2b). This device tends to move the grasped object in the centered position during the closing motion when cable 0 is tightened. Once centered, the object is immobilized. However, contrarily to the grasp exerted by mechanism (a), infinitesimal motions can still occur in this centered configuration. The grasp is 2 nd order form-closed. To make it 1 st order form close, extra nonbackdrivable mechanisms should be added in the transmission of the closing motion of the hand. This aspect will be emphasize in next section while introducing simple design rules to achieve 1 st order form-closure.

\section{Design rules for 1 st order form-closure of underac- tuated hands}

\subsection{The minimum number of unilateral constraints}

As illustrated by these two examples, the introduction of nonbackdrivable mechanisms into the transmission of the closing motion of an underactuated gripper has a direct influence on the stability of the grasp.

In this paper, mathematical formulations that could justify the above assertions are not given (see Krut et al. (2010) to get them). These assertions are based on intuition, and should help us to understand that there is an analogy between the underactuated grasps illustrated in Fig. 2 and the classical grasps illustrated in Fig. 1:

- grasps shown on Fig. 1a and Fig. 2a are both 1st order form-closed, whereas

- grasps shown on Fig. 1b and Fig. 2b are both 2nd order form closed.

This analogy allows understanding how it is possible to extend an important result from classical grasps (exerted by fully actuated grippers) to underactuated grasps: that of the minimum number of contact points necessary for 1 st order form-closure. These works were initiated by Reulaux (1976) and Somov (1900), and later demonstrated by Lakshminarayana (1978), Markenscoff et al. (1990) and Reulaux
(1976). They demonstrate that $d+1$ contacts are necessary to achieve 1st order form-closure of classical grasps:

$n_{\mathrm{c}} \geq d+1$

where $n_{\mathrm{c}}$ is the number of unilateral contact constraints and $d$ is the dimension of the configuration space of the object ( 3 for the planar examples depicted in Fig. 1, 6 in the general case).

By analogy, the following inequality can be written for underactuated grasps:

$n_{\mathrm{k}} \geq g+1$

where $n_{\mathrm{k}}$ is the number of unilateral constraints of the problem and $g$ is the dimension of the grasp configuration space.

This can be stated the following way:

"At least g+1 unilateral constraints are necessary for 1st order form-closure."

The unilateral constraints of the problem are composed (see Krut et al. (2010) for more details) of the unilateral contact constraints and the unilateral constraints imposed by the unidirectional mechanisms (such as worm gears or free wheels). Hence:

$n_{\mathrm{k}}=n_{\mathrm{u}}+n_{\mathrm{c}}$

with $n_{\mathrm{u}}$ the number of unilateral constraints imposed by the unidirectional mechanisms, and $n_{\mathrm{c}}$ is the number of unilateral contact constraints.

Additionally, the grasp configuration space is made of the configuration space of the object and the hand considered separately. Hence:

$g=n_{\mathrm{p}}+d$

with $n_{\mathrm{p}}$ the dimension of the hand configuration space, that is, the number of degrees of freedom of the hand, and $d$ is the dimension of the configuration space of the object.

\subsection{The minimum number of unidirectional mechanisms}

Relation (8) can be rewritten as:

$n_{u}+n_{c} \geq n_{p}+d+1$

Considering a hand with as many DOF as phalanxes, and a grasp where each phalanx exerts one contact point with the object including the palm, leads to:

$n_{c}=n_{p}+1$

Equations (11) and (12) enable a simple rule for designing underactuated hands, capable of performing 1st order form-closed grasps: 
Table 1. Characteristics of underactuated robotic hands. The symbol - indicates that there is no underactuation or no non-backdriveable mechanisms depending on the column.

\begin{tabular}{|c|c|c|c|c|c|c|c|}
\hline Name & $\begin{array}{l}\text { No. of } \\
\text { DOF }\end{array}$ & $\begin{array}{l}\text { No. of act. } \\
\text { for closure }\end{array}$ & $\begin{array}{l}\text { No. of act. } \\
\text { for config. }\end{array}$ & $\begin{array}{l}\text { Type of underact. } \\
\text { between fingers }\end{array}$ & $\begin{array}{l}\text { Type of underact. } \\
\text { between phalanxes }\end{array}$ & $\begin{array}{l}\text { Non-backdriveable } \\
\text { mechanisms }\end{array}$ & Reference \\
\hline Soft Gripper & 18 & 2 & 0 & differential pulleys/cables & differential pulleys/cables & - & Hirose and Umetani (1978) \\
\hline Hand of the LMS & 9 & 1 & 0 & differential with cables & differential pulleys/cables & - & Champeau (1991) \\
\hline TU Delft Hand & 5 & 1 & 0 & differential with cables & differential pulleys/cables & - & De Visser and Herder (2000) \\
\hline Barrett Hand & 7 & 3 & 1 & - & self-locking & 6 wheels and worm drives & Townsend (2000) \\
\hline TBM Prosthesis & 15 & 1 & 0 & compliant & differential four-bar & - & Dechev et al. (2001) \\
\hline Southampton Hand Prosthesis & 15 & 4 & 2 & - & differential four-bar & 6 wheels and worm drives & Light and Chappell (2000) \\
\hline Lopez Hand & 9 & 1 & 0 & self-locking & differential pulleys/cable & 9 bracing devices & Lopez (2001) \\
\hline MARS & 12 & 3 & 3 & - $\quad$ - & differential four-bar & - & Laliberté et al. (2002) \\
\hline SARAH & 10 & 1 & 1 & self-locking & differential four-bar & 3 wheels and worm drives & Laliberté et al. (2002) \\
\hline Laval Univ. Pneumatic Hand & 10 & 3 & 1 & - & differential four-bar & 3 motorised anti-return valves & Laliberté and Gosselin (2003) \\
\hline RTR II & 9 & 1 & 1 & compliant & differential pulleys/cables & 1 wheel and worm drive & Massa et al. (2002) \\
\hline SPRING Hand & 8 & 1 & 0 & compliant & differential pulleys/cables & 1 wheel and worm drive & Carrozza et al. (2004) \\
\hline RTR IV & 14 & 1 & 0 & compliant & differential with cables & - & Carrozza et al. (2005) \\
\hline Nasser Prosthesis & 15 & 1 & 0 & self-locking & differential four-bar & 4 wheels and worm drives & Nasser et al. (2006) \\
\hline Cyberhand & 16 & 5 & 1 & - & differential pulleys/cables & - & Cipriani et al. (2006) \\
\hline Laval Univ. Prosthesis & 17 & 1 & 0 & differential pulleys/cables & differential pulleys/cables & - & Birglen and Gosselin (2006) \\
\hline SDM Hand & 8 & 1 & 0 & differential pulleys/cables & differential with cables & - & Dollar and Howe (2007) \\
\hline iLimb & 11 & 5 & 1 & - & differential with tendons & 5 wheels and worm drives & Gow (2007) \\
\hline Southampton End Effector Hand & 8 & 1 & 0 & differential four-bar & differential four-bar & - & Crowder (1991) \\
\hline
\end{tabular}

"When considering that each phalanx exerts one contact point with the object including the palm, at least $d$ unidirectional mechanisms are required for an underactuated hand to be capable of performing 1st order form-closed grasps."

The TWIX hand, proposed by the authors, was designed according to this simple design rule (Bégoc et al., 2007). Note that for the grasps illustrated in Fig. 2, as there is no palm and as the dimension of the configuration space is $d=1$, at least 2 unidirectional mechanisms are required for an underactuated hand to perform 1st order form-closed grasps. This witnesses why the grasp illustrated in Fig. 2a., performed by an underactuated hand having 2 unidirectional mechanisms, is 1 st order form closed, while the one of Fig. 2b., performed by an underactuated hand having only one unidirectional mechanism (the actuator), is only 2 nd order form-closed.

\subsection{Discussion and analysis of some underactuated hands}

Table 1 lists the main characteristics of various existing underactuated hands. For each hand, the table lists the number of dof, the number of actuators used to drive the closing/opening motion of the hand and the number of actuators used to modify the configuration of the hand. The type of underactuation used for the finger inputs and the phalanx inputs is listed separately, often being of different types. Finally, the number and type of the non-backdrivable mechanisms introduced in the mechanical transmission of the closing motion of the hand are indicated.

One of the major limitations of underactuated hands lies in the ejection phenomenon described in Laliberté et al. (2002). As already mentioned, in certain configurations of the finger, the force exerted by certain phalanxes on the object must be negative to achieve static equilibrium of the finger. Because the contact forces are unilateral, this equilibrium cannot be attained, which causes a backward motion of the corresponding phalanxes. Introducing a unidirectional mechanism in the transmission of the closing motion of each phalanx prevents any backward motion of the phalanx and consequently eliminates the ejection phenomenon. This explains why a large number of underactuated hands described in Table 1 use nonbackdrivable mechanisms without, however, justifying it.

On the basis of the design rule introduced in the previous section, let us review the existing underactuated hands listed in Table 1 It can be noted that, out of the 19 hands, only 3 integrate at least six unidirectional mechanisms: the Lopez hand, the Southampton hand, and the Barrett hand. The other hands in the table cannot achieve 1st order formclosed grasps. Of course they are capable of performing stable grasps, but exhibiting other types of stability such as higher order form-closure or force-closure.

Following this simple rule, when designing underactuated hands, may improve the quality of the grasp in some circumstances. For us, the best proof is that the Barrett hand, that satisfies this simple rule, is the only one that encountered success in industry. However the systematic adoption of that design rule can lead to an increased complexity of the overall mechanical structure of the hand, thus compromising the main appeal of underactuation, that is, simplicity, low cost and so on.

\section{Conclusions}

In this paper, the inadequation of the definition of formclosure, as largely accepted in the literature, with the case of underactuated grasps has been pointed out. The definition is based on the assumption that contact points are fixed in space; this hypothesis is no longer true when the grasp is ex- 
erted by an underactuated hand. Therefore, a more general definition has been introduced, which consists in verifying that the system formed by the hand and the object is immobilized by a set of kinematic constraints. Among these constraints are the contact constraints to which the constraints imposed by unidirectional mechanisms have been added. Indeed, it has been shown, using several simple examples, that unilateral mechanisms have a relevant influence on the stability of the grasp. Hence, a new classification of underactuated hands has been proposed, based on the study of the expression of contact forces. Numerous existing underactuated hands use unilateral mechanisms, without justifying it.

Finally, this study allowed us to state a simple design rule on the minimum number of unidirectional mechanisms to be introduced into the transmission of the closing motion of the fingers of an underactuated hand, so that it is capable of producing 1st order form-closed grasps.

Edited by: J. L. Herder

Reviewed by: two anonymous referees

\section{References}

Ambrose, R. O., Aldridge, H., Askew, R. S., Burridge, R. R., Bluethmann, W., Diftler, M., Lovchik, C., Magruder, D., and Rehnmark, F.: Robonaut: NASA's space humanoid, IEEE Intell. Syst., 15(4), 57-63, 2000.

Bégoc, V., Durand, C., Krut, S., Dombre, E., and Pierrot, F.: On the Form-Closure Capability of Robotic Underactuated Hands, Proc. of ICARCV 2006: 9th International Conference on Control, Automation, Robotics and Vision, Grand Hyatt, Singapore, 5-8 December 2006, 2011-2018, 2006.

Bégoc, V., Krut, S., Dombre, E., Durand, C., and Pierrot, F.: Mechanical design of a new pneumatically driven underactuated hand, Proc. of IEEE Int. Conf. on Robotics and Automation (ICRA'07), Roma, Italy, 927-933, 2007.

Bicchi, A.: On the closure properties of robotic grasping, Int. J. Robot. Res., 14(4), 319-334, 1995.

Birglen, L. and Gosselin, C.: Force analysis of connected differential mechanisms: application to grasping, Int. J. Robot. Res., 25(10), 1033-1046, 2006.

Birglen, L., Laliberté, T., and Gosselin, C.: Underactuated Robotic Hands, Springer-Verlag, ISBN 978-3-540-77458-7, 2008.

Butterfass, J., Grebenstein, M., Liu, H., and Hirzinger, G.: DLRhand II next generation of a dextrous robot hand, Proc. of IEEE Int. Conf. on Robotics and Automation (ICRA'01), Seoul, Korea, 21-26 May 2001, 109-120, 2001.

Carrozza, M., Suppo, C., Sebastiani, F., Massa, B., Vecchi, F., Lazzarini, R., Cutkosky, M., and Dario, P.: The SPRING hand: development of a self-adaptive prosthesis for restoring natural grasping, Autonomous Robots, 16(2), 125-141, 2004.

Carrozza, M., Cappiello, G., Stellin, G., Zaccone, F., Vecchi, F., Micera, S., and Dario, P.: A cosmetic prosthetic hand with tendon driven under-actuated mechanism and compliant joints: ongoing research and preliminary results, Proc. of IEEE Int. Conf. on Robotics and Automation (ICRA'05), Barcelona, Spain, 1822 April 2005, 2672-2677, 2005.
Champeau, H.: Conception et réalisation d'une main mécanique anthropomorphe en vue de son implantation sur le robot manus, M.S. report, Poitiers University of Sciences, France, 1991.

Cheong, J.: Immobilizing grasps for two- and three-dimensional objects, Ph.D. Thesis, Utrecht University, The Netherlands, 2006.

Cipriani, C., Zaccone, F., Stellin, G., Beccai, L., Cappiello, G., Carrozza, M. C., and Dario, P.: Closed-loop controller for a bioinspired multi-fingered underactuated prosthesis, Proc. of IEEE Int. Conf. on Robotics and Automation (ICRA'06), Orlando, Florida, USA, 15-19 May 2006, 2111-2116, 2006.

Crowder, R. M.: An anthropomorphic robotic end effector, Robot. Auton. Syst., 7(4), 253-268, 1991.

De Visser, H. and Herder, J.: Force directed design of a voluntary closing hand prosthesis, J. Rehabil. Res. Dev., 37(3), 261-271, 2000.

Dechev, N., Cleghorn, W. L., and Naumann, S.: Multiple finger, passive adaptive grasp prosthetic hand, Mech. Mach. Theory, 36(10), 1157-1173, 2001.

Dollar, A. M. and Howe, R. D.: Simple, robust autonomous grasping in unstructured environments, Proc. of IEEE Int. Conf. on Robotics and Automation (ICRA'07), Roma, Italy, 10-14 April 2007, 4693-4700, 2007.

Gow, D. J.: Prostheses with Mechanically Operable Digit Members, Touch Emas LTD., Int. patent WO2007063266 (A1), 7 June 2007.

Hirose, S. and Umetani, Y.: The development of soft gripper for the versatile robot hand, Mech. Mach. Theory, 351-359, 1978.

IFToMM website: http://www.iftomm.3me.tudelft.nl/, version 2.3, April 2010.

Kaneko, K., Harada, K., and Kanehiro, F.: Development of multifingered hand for life-size humanoid robots, Proc. of IEEE Int. Conf. on Robotics and Automation (ICRA'07), Roma, Italy, 1014 April 2007, 913-920, 2007.

Krut, S.: A force-isotropic underactuated finger, Proc. of IEEE Int. Conf. on Robotics and Automation (ICRA'05), Barcelona, Spain, 18-22 April 2005, 2314-2319, 2005.

Krut, S., Bégoc, V., Dombre, E., and Pierrot, F.: Extension of the Form-Closure Property to Underactuated Hands, IEEE T-RO: Transaction on Robotics, 26(5), 853-866, October 2010.

Lakshminarayana, K.: Mechanics of form closure, ASME Technical paper 78-DET-32, 2-8, 1978.

Laliberté, T. and Gosselin, C.: Actuation system for highly underactuated gripping mechanism, US patent no. US6.505.870, 2003.

Laliberté, T., Birglen, L., and Gosselin, C.: Underactuation in robotic grasping hands, Japanese Journal of Machine Intelligence and Robotic Control, Special Issue on Underactuated Robots, 4(3), 77-87, 2002.

Light, C. and Chappell, P.: Development of a lightweight and adaptable multiple-axis hand prosthesis, Med. Eng. Phys., 22(10), 679-684, 2000.

Lopez, G.: Préhenseur anthropomorphe à serrage automatique, French patent no. FR2.822.404, 2001.

Luo, M., Mei, T., Wang, X., and Yu, Y.: Grasp Characteristics of an Underactuated Robot hand, Proc. of IEEE Int. Conf. on Robotics and Automation (ICRA'04), New Orleans, LA, USA, 26 April1 May 2004, 2236-2241, 2004.

Markenscoff, X., Ni, L., and Papadimitriou, C. H.: The geometry of grasping, Int. J. Robot. Res., 9(1), 61-74, 1990. 
Massa, B., Roccella, S., Carrozza, M. C., and Dario, P.: Design and development of an underactuated prosthetic hand, Proc. of IEEE Int. Conf. on Robotics and Automation (ICRA'02), Washington, USA, 11-15 May 2002, 3374-3379, 2002.

Nasser, S., Rincon, D., and Rodriguez, M.: Design of an anthropomorphic underactuated hand prosthesis with passive-adaptive grasping capabilities, Florida Conf. on Recent Advances in Robotics and Robot Showcase, Miami, Florida, USA, 25-26 May 2006.

Reulaux, F.: The kinematics of machinery, MacMillan, NY, 1976.

Rimon, E. and Burdick, J. W.: On force and form closure for multiple finger grasps, Proc. of IEEE Int. Conf. on Robotics and Automation (ICRA'96), Minneapolis, MN, USA, 22-28 April 1996, 1795-1800, 1996.
Somov, P.: Über Gebiete von Schraubengeschwindigkeiten eines starren Korpers bei verschiedener Zahl von Stutzflachen, Zeitschrift für Mathematik und Physik, 45, 245-306, 1900.

Townsend, W.: The Barrett Hand grasper - programmably flexible part handling and assembly, Ind. Robot, 27(3), 181-188, 2000.

Ueda, J., Ishida, Y., Kondo, M., and Ogasawara, T.: Development of the NAIST-hand with vision-based tactile fingertip sensor, Proc. of IEEE Int. Conf. on Robotics and Automation (ICRA'05), Barcelona, Spain, 18-22 April 2005, 2332-2337, 2005.

Van der Stappen, A., Wentink, C., and Overmars, M.: Computing immobilizing grasps of polygonal parts, Int. J. Robot. Res., 19(5), 467-479, 2000. 